\title{
Genomic testing as a tool to optimise drug therapy
}

\section{SUMMARY}

A person's genetic make-up, including their ethnicity, can affect how they respond to a drug. It can also contribute to drug toxicity and efficacy.

Pharmacogenomic testing is now inexpensive, relatively fast and can enhance patient care.

Pre-emptive tests for azathioprine and abacavir are subsidised by Medicare.

A national regulatory system including standardised reporting and guidelines for interpreting test results is urgently needed. Improved education for GPs and pharmacists at postgraduate and undergraduate levels is also needed.

\section{Introduction}

The effectiveness and safety of a drug dose are influenced by several patient factors including age, disease, lifestyle and concomitant drugs. Genetic factors can also play a critical role and in some cases genetic testing has become part of treatment guidelines. Tests are performed on patients' DNA from blood, saliva or buccal samples. Some of these tests are subsidised by Medicare, but many are not (see Table).

Currently most testing to optimise therapy is done to prevent severe and life-threatening adverse effects. Twenty years ago, the technology to perform rapid turnaround testing was very limited, time consuming, expensive and insufficiently specific. However, modern genetic technology makes testing feasible as it requires a very small amount of DNA, it is cheap (less than $\$ 1$ per variant) and provides results quickly. The terminology has changed from the original pharmacogenetics to pharmacogenomics. This arose with changes in technology allowing all genes to be sequenced in a single test.

\section{Why are some drugs subject to testing?}

Genetic testing can be used as a tool to optimise drug therapy. Genes control the production of proteins that metabolise and excrete drugs and transport them to their site of action in the body. Proteins are also the targets of drugs. Genes are polymorphic, meaning they have a number of variants that can lead to loss (the most common) or gain of function, or have minimal or no effect on protein function. In most cases, the clinical relevance is minor and genetic testing cannot be justified. However, for a few drugs, testing prevents life-threatening reactions in susceptible people and is recommended in routine practice.

In some ethnic populations, the frequencies of specific variant alleles are substantially different from those of Caucasians. This may affect the way patients respond to drugs such as thiopurines, allopurinol and carbamazepine, and increase their risk of severe adverse reactions.

\section{Allopurinol}

People with the human leukocyte antigen HLA-B*5801 allele given allopurinol can develop a drug reaction with eosinophilia and systemic symptoms (DRESS) ${ }^{1}$ and Stevens-Johnson syndrome or toxic epidermal necrolysis which are severe and life-threatening. These reactions are more likely to occur within the first two months of therapy. Although the carriage of the HLA-B*5801 allele has a much higher frequency in people with Asian ancestry compared with Caucasians (5-15\% vs $\leq 6 \%$ ), it is still represented in European and African populations. In Australia, the carriage rate is about $3 \%$.

\section{Carbamazepine}

HLA-B*1502 screening may be warranted to prevent Stevens-Johnson syndrome or toxic epidermal necrolysis associated with carbamazepine. Carriage of HLA-B*1502 is prevalent in South-East Asian and South Asian populations (10-20\%) but rare in European populations (<0.1\%). In 2007 the US Food and Drug Administration mandated notification of this risk in the product information for carbamazepine with a specific recommendation for HLA-B*1502 screening in South-East Asian populations.

\section{Andrew A Somogyi \\ Professor \\ Discipline of Pharmacology Adelaide Medical School University of Adelaide \\ Professor \\ Clinical and Experimental Pharmacology \\ Department of Clinical \\ Pharmacology Royal Adelaide Hospital Adelaide}

\section{Elizabeth Phillips}

Professor

Medicine, Pharmacology,

Pathology, Immunology

and Microbiology

Department of Medicine

Vanderbilt University

Medical Center

Nashville, Tennessee

USA

Professor and Director

Centre for Clinical

Pharmacology and Infectious Diseases Institute for Immunology and Infectious Diseases Murdoch University Perth

\section{Keywords}

abacavir, adverse drug reaction, allopurinol, azathioprine,

carbamazepine, pharmacogenomics, phenytoin

Aust Prescr 2017:40:101-4 http://dx.doi.org/10.18773/ austprescr.2017.027 


\section{Table Pharmacogenomic tests in Australia}

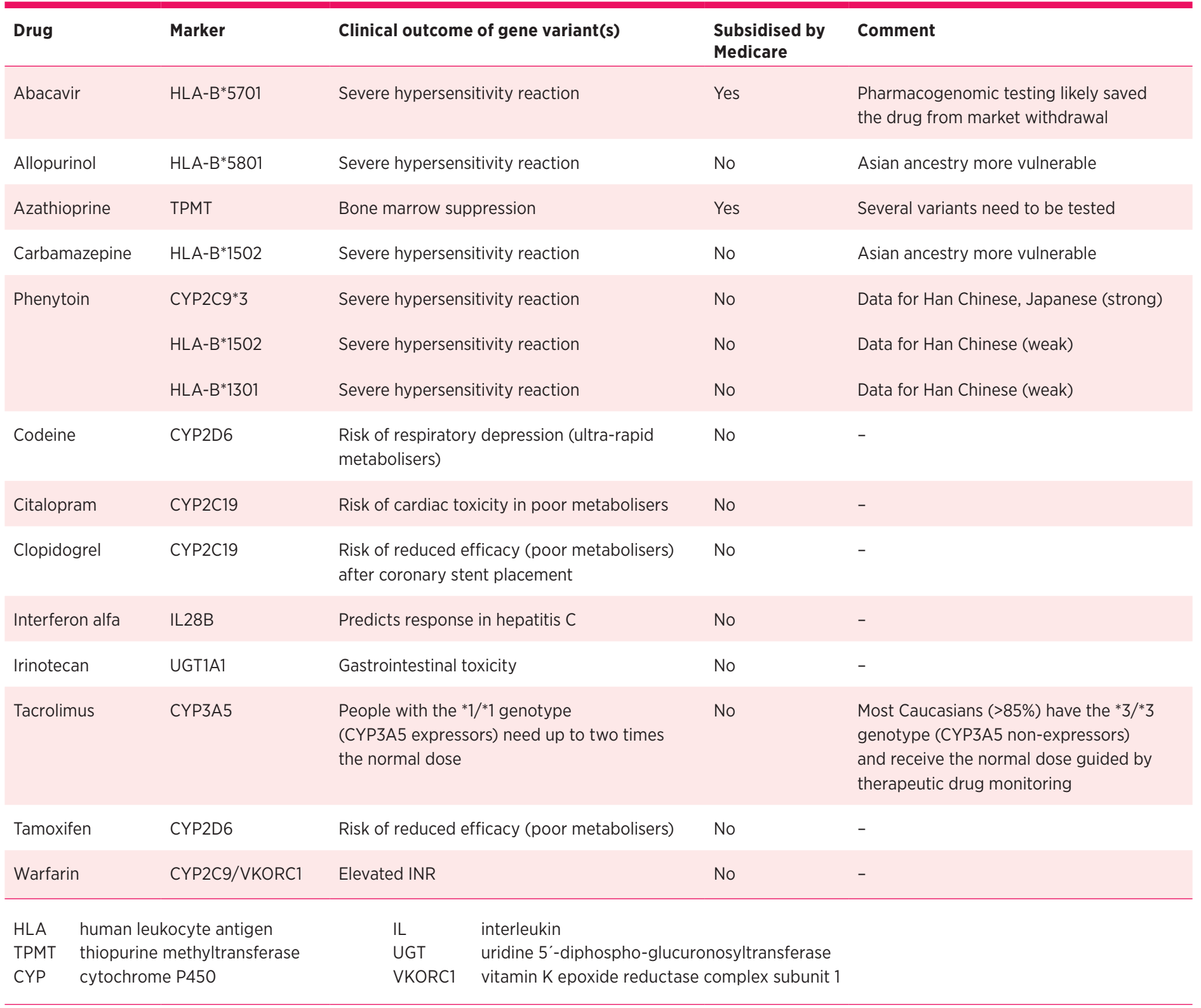

\section{Thiopurine drugs}

Thiopurine drugs are immunosuppressants used in some autoimmune and inflammatory diseases, and blood cancers. They include azathioprine, mercaptopurine and tioguanine (used in some leukaemias). These drugs can cause severe, lifethreatening bone marrow suppression in $1-5 \%$ of patients on standard doses. This is due to a deficiency in thiopurine methyltransferase (TPMT) activity because of variations in the TPMT gene.

TPMT is an enzyme that metabolises thiopurine drugs. About one in 300 people have very low TPMT activity and are susceptible to this severe reaction. Bone marrow toxicity can be avoided if appropriate dosing recommendations are made based on genetic testing (see Table).
The TPMT*3C allele results in low enzyme activity. It has a frequency of almost $10 \%$ in African populations, less than $1 \%$ in Caucasians and is practically nonexistent in South-East Asians.

TPMT genetic testing has been subsidised by Medicare since 2011. Most prescribers (usually hospital-based specialists) will pre-emptively order the test in patients who are likely to be prescribed thiopurine drugs.

\section{Abacavir}

The nucleoside reverse transcriptase inhibitor abacavir is indicated in HIV. A multi-organ severe hypersensitivity syndrome occurs in $5 \%$ of those prescribed abacavir. This syndrome is strongly associated with HLA-B*5701. The absence of this allele has a $100 \%$ negative 
predictive value for abacavir hypersensitivity. Medicare began funding the test in 2009 and it is now routinely performed before abacavir prescription.

\section{Phenytoin}

There is an association between poor metabolisers of phenytoin (with cytochrome P450 (CYP) 2C9*3) and severe cutaneous adverse reactions such as DRESS and Stevens-Johnson syndrome or toxic epidermal necrolysis.

\section{Citalopram}

There is a risk of cardiac toxicity with citalopram in patients who are poor CYP2C19 metabolisers. The product information recommends an initial dose of $10 \mathrm{mg}$ daily during the first two weeks for at-risk patients.

\section{Codeine}

There is an increased risk of respiratory depression with codeine in patients who are CYP2D6 ultra-rapid metabolisers because they rapidly and extensively convert codeine to morphine. This phenotype is mainly due to multiple CYP2D6 gene copies.

\section{What is the evidence for pharmacogenomic testing?}

In most cases, randomised clinical trials of pharmacogenomic testing have not been conducted because of cost, the drugs may be off-patent, recruitment of sufficient patients is difficult given the incidence of the drug reaction is often very low, and a perception that it is unimportant. It is more common for evidence to be based on systematic reviews and meta-analyses, with consensus statements that testing should be done because it improves safety and efficacy.

Given that testing can result in drug selection or dosage modification, it is no different from other dosing recommendations listed in the product information, such as older age, renal and hepatic dysfunctions, and drug interactions, which have rarely been the subject of randomised clinical trials. ${ }^{2}$

A first-of-its-kind trial conducted for abacavir showed that pre-emptive testing for HLA-B*5701 eliminated immunologically confirmed hypersensitivity to abacavir. ${ }^{3}$ Trials of testing for CYP2C9/VKORC1 before starting warfarin had mixed results. Other studies of CYP2C19 testing before clopidogrel therapy had positive results in specific patients.

\section{Direct-to-consumer testing}

Patients can obtain their pharmacogenomic information by accessing international and Australian testing companies either directly or sometimes via a pharmacy. These companies invariably do not have specific patient details (e.g. comorbidities, severity of disease, concomitant drugs) and are limited with what recommendations can be made. The reporting back to the individual or pharmacist can be vague. For example, the report may simply state the genotype (e.g. CYP2D6*4/*4) with a simple statement of 'poor metaboliser'.

Most companies do not test the HLA alleles associated with severe adverse drug effects. Also, the drugs they recommend for dose adjustment may have been insufficiently evaluated for pharmacogenomic testing to be of value. At issue is the regulatory environment of pharmacogenomic testing and what decision support systems are in place to help doctors and patients.

\section{Regulation and education}

In Australia, the regulation of pharmacogenomic testing seems to come under the umbrella of medical devices rather than medicines at the Therapeutic Goods Administration. An overarching regulatory framework involving national regulatory bodies, with advice from professional societies, is needed to resolve critical issues. These include the need to provide broad guidance on what variant alleles should be tested, laboratory validation of the test and, more importantly, the interpretation of the test and guidelines for changing the drug or its dose. Ideally these should not be laboratory specific as is the case now. A national regulatory consensus and reporting template is warranted, so that for example a GP in Queensland will receive the same report and recommendation as a GP in Tasmania.

As genetic testing moves into mainstream medicine, there remains a clear need to improve education for GPs, specialists, pharmacists, medical students and other healthcare professionals. ${ }^{4}$

\section{Conclusion}

Genomic testing to optimise drug therapy is a new diagnostic tool that will increase in frequency as new discoveries are made. It will have implications for physicians, and increasingly for GPs, who coordinate patient care. In particular, pharmacogenomic testing to reduce the potential for drug-induced severe and life-threatening toxicity has immediate implications, particularly for specific ethnic groups at greater risk. ${ }^{5}$ More drug tests will become available on Medicare once the evidence becomes established.

National regulation of pharmacogenomic testing with specific reporting and interpretation templates is needed before direct-to-consumer testing by multiple 
<ustralian Prescriber

providers creates confusion for patients and their health professionals. ${ }^{6}$ Pre-emptive testing, companion diagnostics, point-of-care testing and decision support systems to assist doctors, patients and pharmacists need to be quickly addressed. Education for doctors and pharmacists is necessary to ensure that patients obtain their optimal pharmacotherapy based on precision medicine. $<$
Andrew Somogyi is a co-holder of a patent for a perhexiline formulation. He receives funding from the National Health and Medical Research Council (NHMRC).

Elizabeth Phillips is a co-director of IIID Pty Ltd that holds a patent for HLA-B*5701 testing. She receives funding from the NHMRC, Australian Centre for HIV and Hepatitis Virology Research, and National Institutes of Health (1P50GM115305-01, 1R01A/103348-01, 1P30A/110527-01A1).

\section{REFERENCES}

1. Mugwagwa AN, Fischer R, Zailan I. HLA-B*5801: a genetic susceptibility to allopurinol-induced DRESS. Med J Aust 2016;204:159-60. http://dx.doi.org/10.5694/mja15.01113

2. Pirmohamed $M$, Hughes DA. Pharmacogenetic tests: the need for a level playing field. Nat Rev Drug Discov 2013;12:3-4. http://dx.doi.org/10.1038/nrd3921

3. Mallal S, Phillips E, Carosi G, Molina JM, Workman C, Tomazic J, et al.; PREDICT-1 Study Team. HLA-B*5701 screening for hypersensitivity to abacavir. N Engl J Med 2008:358:568-79. http://dx.doi.org/10.1056/NEJMoa0706135

4. Nickola TJ, Green JS, Harralson AF, O'Brien TJ. The current and future state of pharmacogenomics medical education in the USA. Pharmacogenomics 2012;13:1419-25. http://dx.doi.org/10.2217/pgs.12.113
5. Relling MV, Evans WE. Pharmacogenomics in the clinic Nature 2015;526:343-50. http://dx.doi.org/10.1038/ nature15817

6. Caudle KE, Dunnenberger HM, Freimuth RR, Peterson JF, Burlinson JD, Whirl-Carillo M, et al. Standardizing terms for clinical pharmacogenetic test results: consensus terms from the Clinical Pharmacogenetics Implementation Consortium (CPIC). Genet Med 2017;19:215-23. http://dx.doi.org/10.1038/ $\operatorname{gim} .2016 .87$

\section{FURTHER READING}

Liew D, Keith C, Booth J, Perera D. Medicinal mishap: Fatal azathioprine toxicity. Aust Prescr 2017;40:109. http://dx.doi.org/ 10.18773 /austprescr.2017.035 